\title{
Petri Based Recurrent Fuzzy Neural Control for SY-II Remote Operated Vehicle
}

\author{
Huang Hai, Wan Lei, Zhang Guocheng, Pang Yongjie \\ Key Laboratory of Science and Technology for National Defense of Autonomous Underwater Vehicle \\ Harbin Engineering University \\ Harbin, China
}

\begin{abstract}
Recurrent fuzzy neural network is widely applied in many areas because it combines the advantages of low level learning and high level reasoning. In considering the complicated factors and requirements for the Remote Operated Vehicle(ROV) control, petri network has been introduced to design a dynamic controller for underwater robot. It intends to reduce the computation burdens during network parameters learning. The gradient descent method has been used for online training. In order to guarantee its convergence, we have used the discrete Lyapunov function to determine its learning rate. The tank experiments have proved that the controller can adjust control quantity to reduce caculation and present strong advantages in the ROV robustness control.
\end{abstract}

Keywords-Remote Operated Vehicle; Petri Network(PN); Recurrent Fuzzy Neural Network(RFNN)

\section{INTRODUCTION}

With aboundunt power supply, ROV can exchange a great deal of information with its surface work station. It can be utilized broadly in marine exploration and resource exploitation[1,2]. To realize object detection and oceania task, accurate motion control must be fundamental. However, it is very difficult to construct a precise dynamic model for 6-DOF ROV underwater motion, not only because the sea environment is complicated and unknown, but the ROV itself is the strongly nonlinear and coupling[3,4].

In the combination with $\mathrm{S}$ surface control and fuzzy concept, reference[5] not only improved the control effectiveness but simplified the controller design. But this method is based on proportion and integration priciple, it does not possess the capacity of self-learning. The adjustment of its controller parameters depends completely on researcher's experience. In consideration with strong nonlinearity and coupling characters of underwater robots, Neural Network (NN) is good at self-learning to adapt to environment change[6,7]. However vibration may prone to happen, because the NN learning is usually lagging out. In compare with Back Propagation (BP) Neural Network, Recurrent Neural Network (RNN) can deal with dynamic change much better[8,9]. On the other hand, Fuzzy Neural Network (FNN) is widely applied in the control of Underwater robots aboard, because it combines low level learning with high level reasoning[10,11]. Apparently, taking dynamic feedback as memory element, RFNN possesses advantages of the two, but with complicated calculation[12].

Recently, Petri-Network(PN) has become an effective tool to model, control, analyse and optimize. In order to reduce calculation and improve the effectiveness, we introduce PN in the designation of RFNN to solve the ROV control problems such as strong nonlinearity and unknow oceania environment .

\section{SY-II ROV AND ITS CONTROL STRUCTURE}

SY-II ROV (Figure 1) is an open frame underwater robot. It is equipped with a depth guage, magnetic compass, two main thrusters, two side thrusters and two vertical thrusters. If we establish absolute reference frame $E-\xi \eta \zeta$ and relative reference frame G-xyz and neglect roll, 5-DOF motion model can be obtained as:

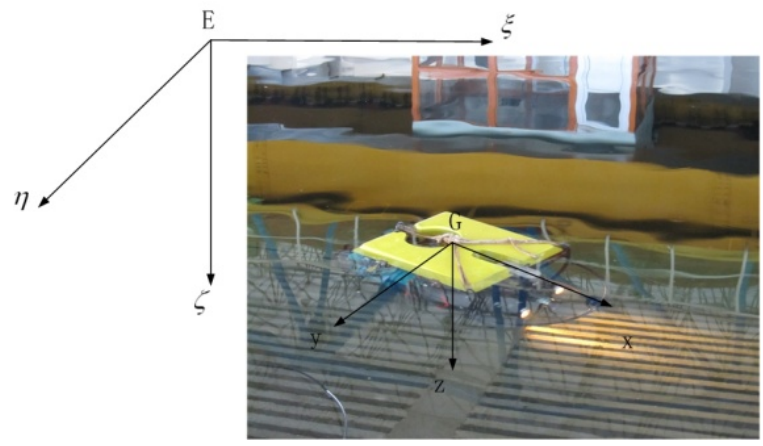

Figure 1. SY-II ROV

$$
\left\{\begin{array}{c}
m\left[(\dot{u}-v r+w q)-x_{G}\left(q^{2}+r^{2}\right)+y_{G} \dot{r}+z_{G} \dot{q}\right]=X \\
m\left[(\dot{v}+u r)-y_{G} r^{2}+z_{G} q r+x_{G} \dot{r}\right]=Y \\
m\left[(\dot{w}-u q)-z_{G} q^{2}-x_{G} \dot{q}+y_{G} r q\right]=Z \\
I_{y} \dot{q}+m \cdot\left[z_{G} \cdot(\dot{u}+w q-v r)-x_{G} \cdot(\dot{w}-u q)\right]=M \\
I_{z} \dot{r}+m \cdot\left[x_{G} \cdot(\dot{v}+u r)-y_{G} \cdot(\dot{u}-v r)\right]=N
\end{array}\right.
$$

where $m$ is the mass of robot; $x_{G}, y_{G}, z_{G}$ are the gravity centers of the robot; $I_{x}, I_{y}, I_{z}$ are the moments of inertia in associated with axes $G x, G y, G z$ respectively; $u, v, w, q, r$ are velocities and angular velocities of the $5 \mathrm{DOF}$ respectively; $X, Y, Z, M, N$ are torques of 5-DOF respectively. Thus the hydrodynamic model is obtained: 


$$
\begin{aligned}
& M(\vec{q}) \ddot{\vec{q}}+C(\dot{\vec{q}}) \dot{\vec{q}}+D(\dot{\vec{q}}) \dot{\vec{q}}+g(\vec{x})=\vec{\tau} \\
& \dot{\vec{x}}=Q \dot{\vec{q}}
\end{aligned}
$$

where $\dot{\vec{q}}=\left[\begin{array}{lllll}u & v & w & q & r\end{array}\right]^{T}, \mathrm{M}$ is the $5 \times 5$ inertia matrix, $\vec{x}=\left[\begin{array}{lllll}x & y & z & \theta & \psi\end{array}\right]^{T}$ is the $5 \times 1$ position vector according to absolute reference frame. $C(\dot{\vec{q}})$ is the $5 \times 1$ vector of centrifugal and Coriolis terms, $D(\dot{\vec{q}})$ is resistance matrix, $g(\vec{x})$ is gravity, $\vec{\tau}$ is the input force vector of thrusters and torques from the controller.

Figure 2. illustrates SY-II's control structure block diagram. The desired forces/torques can be computed by the controller according to the objective values and the the sensors feedback. The depth is obtained from pressure sensor, and SY-II pitch, roll, and heading are measured by the magnetic sensor. The controller in the diagram is PN based RFNN.

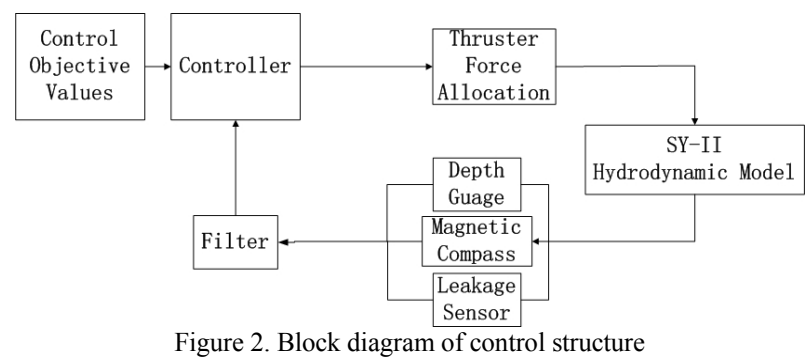

\section{CONTROLler Design}

\section{A.The Controller}

Figure.3 is the 5-layer block diagram of PN based RFNN. It includes input layer, membership layer, petri layer, rule layer and output layer. The feedback connection is realized through reccurent feedback at membership layer. The propagation fuction in each layer are issued as follows.

1). The first layer is input layer. Its output node is expressed $\quad$ as: $\quad y_{i}^{(1)}=x_{i}^{(1)} \quad, \quad i=1,2,3 \ldots n_{i}$ (2) where $x_{i}^{(1)}$ and $y_{i}^{(1)}$ are the input and output of the ith node in this layer, $n_{i}$ is the number of input variables.

2). The second layer is membership layer. Each node in this layer acts as a membership function. The input of this layer is expressed as: $x_{i}^{(2)}(n)=y_{i}^{(1)}(n)+\eta_{i j}(n-1) \theta_{i j}$

where $\theta_{i j}$ denotes the weight of the self-feedback loop, $\mathrm{n}$ is the iteration times, $\eta_{i j}(n-1)$ represents the last time output of $2^{\text {nd }}$ layer. The output of $2^{\text {nd }}$ layer is defined with Gaussian membership functions as:

$y_{i j}^{(2)}=\exp \left(-\frac{\left(x_{i}^{(2)}-m_{i j}\right)^{2}}{\left(\sigma_{i j}\right)^{2}}\right), j=1,2, \ldots, n_{j}$

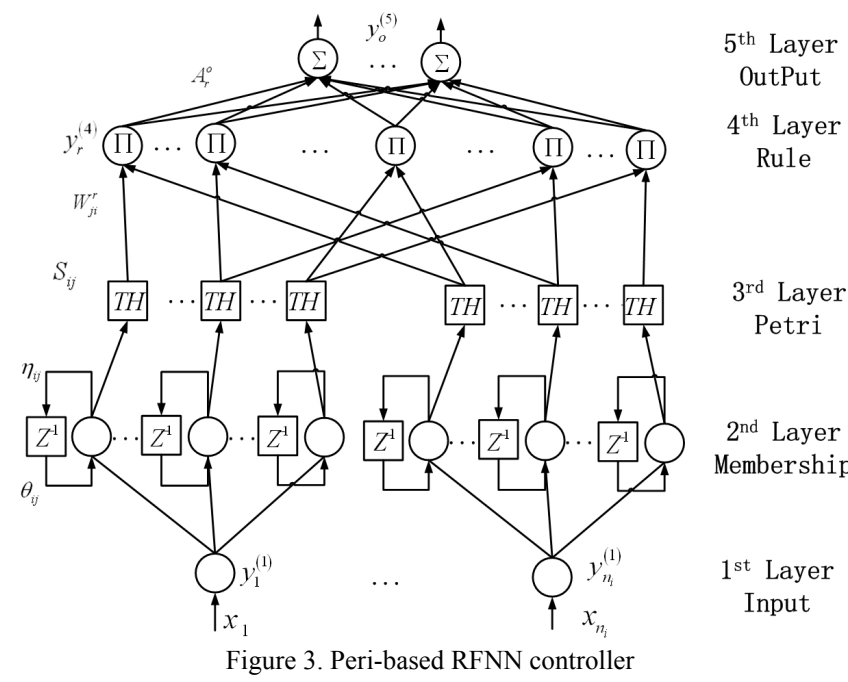

where $m_{i j}$ and $\sigma_{i j}$ are the mean and standard deviation of the Gaussian function respectively. $n_{j}$ is the number of the linguistic variables associated with each input.

3 ). The third layer is Petri layer whose purpose is to produce threshold by using competition laws to determine the training requirements:

$S_{i j}= \begin{cases}1 & y_{i j}^{(2)} \geq T H \\ 0 & y_{i j}^{(2)}<T H\end{cases}$

where $S_{i j}$ is the switch and $T H$ is a dynamic threshold value in corresponding with errors to be determined in III B.

4). The $4^{\text {th }}$ layer is rule layer, which multiplies the input variables to produce output:

$$
y_{r}^{(4)}= \begin{cases}0 & T H=0 \\ \prod_{i=1}^{n_{i}} W_{j i}^{r} y_{i j}^{(2)} & T H=1\end{cases}
$$

where $W_{j i}^{4}$ is the weights between the $3^{\text {rd }}$ layer and the $4^{\text {th }}$ layer, $r=1,2, \ldots, n_{r}$ is the total rule numbers.

5 . The $5^{\text {th }}$ layer is output layer:

$y_{o}^{(5)}=\sum_{r=1}^{n_{r}} A_{r}^{o} y_{r}^{(4)}$ 
where $A_{r}^{o}$ is the output action strength with respect to the rth rule.

\section{B. Strategy for Online Learning}

The core of learning strategy is to obtain a gradient vector so that each element can be defined as the derivative of an energy function. SY-II ROV can be controlled with auto-depth and auto-heading because it is equipped with depth guage and magnetic sensor. This paper will deduce the algorithm from depth and heading control, motion control of other DOFs can be obtained similarly.

First of all, we define the energy function $\mathrm{E}$ as:

$$
\begin{aligned}
& E=\frac{1}{2}\left[\left(h_{d}-h\right)^{2}+\left(\dot{h}_{d}-\dot{h}\right)^{2}+\left(\psi_{d}-\psi\right)^{2}+\left(\dot{\psi}_{d}-\dot{\psi}\right)^{2}\right] \\
& =\frac{1}{2}\left(e_{d h}^{2}+\dot{e}_{d h}^{2}+e_{d \psi}^{2}+\dot{e}_{d \psi}^{2}\right) \\
& 8
\end{aligned}
$$

where $h_{d}$ is desired diving depth, $\psi_{d}$ is desired heading, $h$ is the real diving depth, $\psi$ is the real heading, $e_{d h}=h_{d}-h$ is current depth error, $e_{d \psi}=\psi_{d}-\psi$ is current heading error, $\dot{h}_{d}, \dot{h}, \dot{\psi}, \dot{\psi}_{d}, \dot{e}_{h}$ and $\dot{e}_{\psi}$ are the derivative of $h_{d}, h, \psi, \psi_{d}, e_{d h}$ and $e_{d \psi}$. TH of equation (5) will be defined through the following equation:

$$
T H=\frac{\partial \exp (-\delta E)}{1+\exp (-\delta E)}
$$

where $\partial$ and $\delta$ are positive constants to coordinate TH which will be larger if the error is smaller.

In the output layer the error term to be propagated is:

$\beta=\left(\frac{\partial E}{\partial} \frac{\partial e_{d h}}{\partial h} \partial h \quad \partial E \partial e_{d \psi} \partial \psi \quad \partial E \partial \dot{e}_{d h} \partial \dot{h} \quad \partial E \partial \dot{e}_{d \psi} \partial \dot{\psi}\right)$ where $\mu_{m}$ and $\mu_{\sigma}$ are the learning-rate parameters for the $\beta_{o}=-\left(\frac{\partial E}{\partial e_{d h}} \frac{\partial e_{d h}}{\partial h} \frac{\partial h}{\partial y_{o}^{(5)}}+\frac{\partial E}{\partial e_{d \psi}} \frac{\partial d \psi}{\partial \psi} \frac{\partial \psi}{\partial y_{o}^{(5)}}+\frac{\partial E}{\partial \dot{e}_{d h}} \frac{\partial \dot{e}_{d h}}{\partial \dot{h}} \frac{\partial h}{\partial y_{o}^{(5)}}+\frac{\partial E}{\partial \dot{e}_{d \psi}} \frac{\partial d \psi}{\partial \dot{\psi}} \frac{\partial \psi}{\partial y_{o}^{(5)}}\right.$ mean and the standard deviation of the Gaussian function $=e_{d h} \frac{\partial h}{\partial y_{o}^{(5)}}+e_{d \psi} \frac{\partial \psi}{\partial y_{o}^{(5)}}+\dot{e}_{d h} \frac{\partial \dot{h}}{\partial y_{o}^{(5)}}+\dot{e}_{d \psi} \frac{\partial \dot{\psi}}{\partial y_{o}^{(5)}}$

The weight of output layer is updated by the equation (10):

$$
\Delta A_{k}^{o}=-\mu_{\omega} \frac{\partial E}{\partial \omega_{k}^{o}}=\mu_{\omega}\left[-\frac{\partial E}{\partial y_{o}^{(5)}}\right]\left(\frac{\partial y_{o}^{(5)}}{\partial \omega_{k}^{o}}\right)=\mu_{\omega} \beta_{o} A_{r}^{o}
$$

where $\mu_{\omega}$ is the learning-rate parameter of the connecting weights. The propagating error term can be calculated as:

$$
\beta^{(4)}=-\frac{\partial E}{\partial A_{r}^{o}}=\left[-\frac{\partial E}{\partial y_{o}^{(5)}}\right]\left(\frac{\partial E}{\partial A_{r}^{o}}\right)=\left\{\begin{array}{cc}
\beta_{o} A_{k}^{o}, & A_{r}^{o} \neq 0 \\
0, & A_{r}^{o}=0
\end{array}\right.
$$

In the Petri layer, the error term is:

$$
\begin{aligned}
& \beta_{r}^{(3)}=\frac{\partial E}{\partial r_{i j}} \\
& =\left[\frac{\partial E}{\partial y_{o}^{(5)}} \frac{\partial y_{o}^{(5)}}{\partial A_{r}^{o}}\right]\left(\frac{\partial A_{r}^{o}}{\partial y_{i j}^{(2)} r_{i j}} \frac{\partial y_{i j}^{(2)} r_{i j}}{\partial r_{i j}}\right) \\
& =\left\{\begin{array}{l}
\sum_{r} \beta_{r}^{(4)} y_{r}^{(4)}, \quad S_{i j}=1 \\
0,
\end{array}\right. \\
& \text { where } r_{i j}=-\frac{\left(x_{i}^{(2)}-m_{i j}\right)^{2}}{\left(\sigma_{i j}\right)^{2} . \text { Thus he update laws for } m_{i j},} \\
& \sigma_{i j} \text { and } \theta_{i j} \text { are: } \\
& \Delta m_{i j}=-\mu_{m} \frac{\partial E}{\partial m_{i j}} \quad \mu_{m}\left[-\frac{\partial E}{\partial y_{o}^{(5)}} \frac{\partial y_{o}^{(5)}}{\partial r_{i j}} \frac{\partial r_{i j}}{\partial m_{i j}}\right]=\mu_{m} \beta_{r}^{(3)} \frac{2\left(x_{i}^{(2)}-m_{i j}\right)^{2}}{\left(\sigma_{i j}\right)^{2}} y_{i j}^{(2)}(n-1)
\end{aligned}
$$

$$
\Delta \sigma_{i j}=-\mu_{\sigma} \frac{\partial E}{\partial \sigma_{i j}}=\mu_{\sigma} \beta_{r}^{(3)} \frac{2\left(x_{i}^{(2)}-m_{i j}\right)^{2}}{\left(\sigma_{i j}\right)^{3}}
$$

$$
\Delta \theta_{i j}=-\mu_{\theta} \frac{\partial E}{\partial \theta_{i j}}=-\mu_{\theta} \beta_{r}^{(3)} \frac{2\left(x_{i}^{(2)}-m_{i j}\right)}{\left(\sigma_{i j}\right)^{2}} y_{i j}^{(2)}(n-1)
$$
respectively, $\mu_{\theta}$ is the learning-rate parameter for the selffeedback loop.

$$
A_{k}^{o}(n), m_{i j}(n), \sigma_{i j}(n) \text { and } \theta_{i j}(n) \text { are updated }
$$

as:

$$
(\bullet)(n+1)=(\bullet)(n)+\Delta(\bullet)(n)
$$

In order to improve learning speed, $h, \psi, \dot{h}$ and $\dot{\psi}$ can be approximated by their sign functions $\operatorname{sgn}()$.

$\frac{\partial(*)}{\partial y_{o}^{(5)}} \cong \operatorname{sgn}\left(\frac{(*)(n)-(*)(n-1)}{y_{o}^{(5)}(n)-y_{o}^{(5)}(n-1)}\right)$

where $(*)$ represents $h, \psi, \dot{h}$ and $\dot{\psi}$.

\section{Analysis for Convergence}


Values selection for the learning-rate parameters has significant effects on the network performance. In the following, learning-rate parameters are to be analyzed in associated with the convergence of network.

The discrete-type of (8) is:

$$
\begin{aligned}
& E(n+1)=E(n)+\Delta E(n) \\
= & E(n)+\sum_{k=1}^{n_{r}}\left[\frac{\partial E(n)}{\partial A_{k}^{o}} \Delta A_{k}^{o}\right]+\sum_{i=1}^{n_{i}} \sum_{j=1}^{n_{j}}\left[\partial E(n)\left(\frac{\Delta m_{i j}}{\partial m_{i j}}+\frac{\Delta \sigma_{i j}}{\partial \sigma_{i j}}+\frac{\Delta \theta_{i j}}{\partial \theta_{i j}}\right)\right] \\
= & \frac{1}{4} E(n)-\mu_{\omega} \sum_{k=1}^{n_{r}}\left[\frac{\partial E(n)}{\partial y_{o}^{(5)}} \frac{\partial y_{o}^{(5)}}{\partial A_{k}^{o}}\right]^{2} \\
+ & \frac{1}{4} E(n)-\mu_{m} \sum_{i=1}^{n_{i}} \sum_{j=1}^{n_{j}}\left[\frac{\partial E(n)}{\partial x_{i}} \frac{\partial x_{i}}{\partial y_{o}^{(5)}} \frac{\partial y_{o}^{(5)}}{\partial m_{i j}}\right]^{2} \\
& +\frac{1}{4} E(n)-\mu_{\sigma} \sum_{i=1}^{n_{i}} \sum_{j=1}^{n_{j}}\left[\frac{\partial E(n)}{\partial x_{i}} \frac{\partial x_{i}}{\partial y_{o}^{(5)}} \frac{\partial y_{o}^{(5)}}{\partial \sigma_{i j}}\right]^{2} \\
+ & \frac{1}{4} E(n)-\mu_{\alpha} \sum_{i=1}^{n_{i}} \sum_{j=1}^{n_{j}}\left[\frac{\partial E(n)}{\partial x_{i}} \frac{\partial x_{i}}{\partial y_{o}^{(5)}} \frac{\partial y_{o}^{(5)}}{\partial \theta_{i j}}\right]^{2}
\end{aligned}
$$

Thus $\mu_{\omega}, \mu_{m}, \mu_{\sigma}$ and $\mu_{\theta}$ are set as:

$$
\begin{aligned}
& \mu_{\omega}=\frac{E(n)}{4\left[\sum_{k=1}^{n_{y}}\left[\frac{\partial E(n)}{\partial y_{o}^{(5)}} \frac{\partial y_{o}^{(5)}}{\partial \omega_{k}^{o}}\right]^{2}+\varepsilon\right]} \\
& \mu_{m}=\frac{E(n)}{4\left[\sum_{i=1}^{n_{i}} \sum_{j=1}^{n_{j}}\left[\frac{\partial E(n)}{\partial x_{i}} \frac{\partial x_{i}}{\partial y_{o}^{(5)}} \frac{\partial y_{o}^{(5)}}{\partial m_{i j}}\right]^{2}+\varepsilon\right]} \\
& \mu_{\sigma}=\frac{E(n)}{4\left[\sum_{i=1}^{n_{i}} \sum_{j=1}^{n_{j}}\left[\frac{\partial E(n)}{\partial x_{i}} \frac{\partial x_{i}}{\partial y_{o}^{(5)}} \frac{\partial y_{o}^{(5)}}{\partial \sigma_{i j}}\right]^{2}+\varepsilon\right]} \\
& \mu_{\theta}=\frac{E(n)}{4\left[\sum_{i=1}^{n_{i}} \sum_{j=1}^{n_{j}}\left[\frac{\partial E(n)}{\partial x_{i}} \frac{\partial x_{i}}{\partial y_{o}^{(5)}} \frac{\partial y_{o}^{(5)}}{\partial \theta_{i j}}\right]^{2}+\varepsilon\right]}
\end{aligned}
$$

where $\varepsilon$ is a positive constant. Thus (14) can be written as:

$$
\begin{aligned}
& E(n+1) \approx \varepsilon\left(\mu_{\omega}+\mu_{m}+\mu_{\sigma}+\mu_{\theta}\right) \\
& <\frac{E(n)}{4}+\frac{E(n)}{4}+\frac{E(n)}{4}+\frac{E(n)}{4}=E(n)
\end{aligned}
$$

According to (8) and (16), the proposed PN based RFNN controller is convergence.

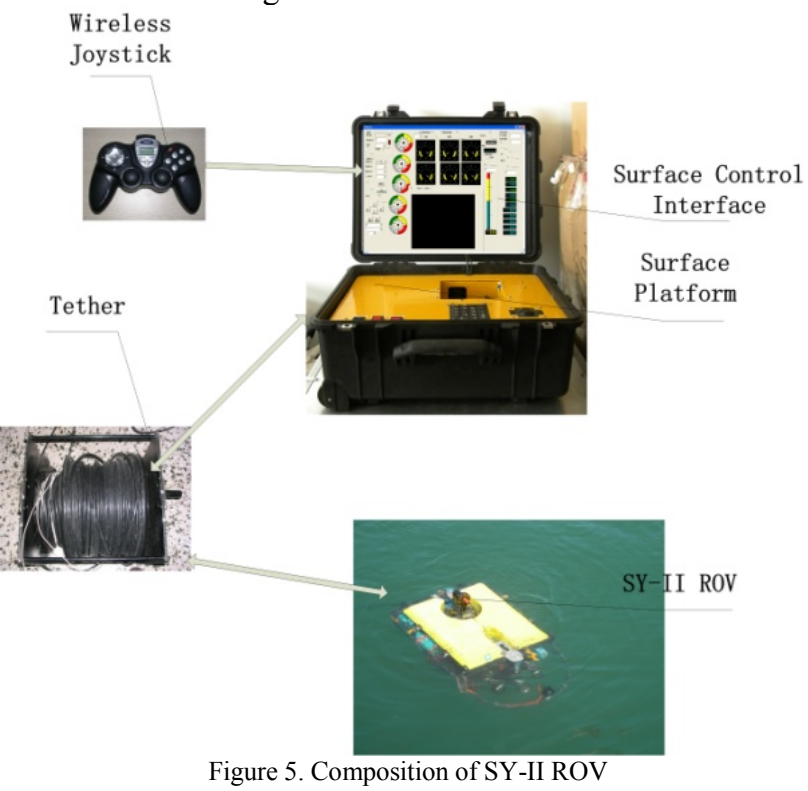

IV. EXPERIMENTS

In order to verify the PN based RFNN controller, experiments has been made in a $50 \times 30 \times 10$ meters pond of the Key Laboratory of Science and Technology for National Defense of Autonomous Underwater Vehicle, Harbin Engineering University.

Figure.5 illustrates SY-II ROV composition. It can not only be manipulated through remote control joystick, but be auto-controlled through surface platform. Communication and power are transmitted through tether between surface platform and underwater vehicle.

In these experiments, we have made comparisons between $\mathrm{PN}$ based RFNN and $\mathrm{S}$ surface control[13,14](which is widely applied in underwater vehicles control). For SY-II, its hard tether provides strong non-linear and random disturbance against auto-control. Particularly being different at different heading, the tether force causes $\mathrm{S}$ surface heading auto-control very difficult. However, PN based RFNN controller can response disturbance very quickly, reduce control errors and display strong robustness.

\section{SUMMARIES}

In order to reduce the computation burdens during recurrent fuzzy neural network parameters learning, petri net has been introduced for the design of dynamic controller of underwater robot. In the online learning strategy, the gradient descent method has been used for online training. 
In order to guarantee its convergence, discrete Lyapunov function has been used to determine its learning rate. The tank experiments have proved that the controller can improve the computation efficiency, reduce control errors, vibration and overshoot, and display strong robustness in the underwater robotic control.

\section{ACKNOWLEDGMENT}

This project is supported by National High Technology Research and Development Program of China (2008AA092301); Postdoctral Science Foundation of Chinese Hei Long-jing province(323630295)

\section{REFERENCES}

[1] Adam J.A. Probing Beneath the Sea [J]. IEEE spectrum,1985,22(4): $55 \sim 64$.

[2] Xu Yu-Ru, Xiao Kun. Technology Development of Autonomous Ocean Vehicle. Acta Automatica Sinica. 33(5): 2007: 518 521.

[3] Li Ye, Zhang Lei, Wan Lei, Liang Xiao. Optimization of S-surface Controller for Autonomous Underwater Vehicle with Immunegenetic algorithm. Journal of Harbin Institute of Technology (New Series). 2008, 15(3):404 410

[4] B. Buckham, F.R. Driscoll and M. Nahon, "Development of a Finite Element Cable Model for Use in Low-Tension Dynamics Simulation," Journal of Applied Mechanics, (71), 2004: 476 485.

[5] F.R. Driscoll, R.G. Lueck, M. Nahon. Development and Validation of a Lumped-mass Dynamics Model of a Deep-sea ROV System. Applied Ocean Research, (22), 2000: 169 182

[6] Tang Xu-Dong, Pang Yong-Jie, Li Ye. Process Neural Control Algorithm for Autonomous Underwater Vehicle. Control Theory and Applications. 26(4): 420 424
[7] Tang Xudong, Pang Yongjie, Li Ye, Qing, Zaibai. A Fuzzy Neural Networks Controller of Underwater Vehicles based on Ant Colony Algorithm. Proceedings of the 27th Chinese Control Conference. Kunming, Yunnan, China. 2008.7: 637 641

[8] R. J. Wai, C. M. Lin, and Y. F. Peng “Adaptive Hybrid Control for Linear Piezoelectric Ceramic Motor Drive Using Diagonal Recurrent CMAC network," IEEE Trans. Neural Netw., 2004, 15(6) : 1491 1506.

[9] S. J. Yoo, Y. H. Choi, and J. B. Park, "Generalized Predictive Control Based on Self-recurrent Wavelet Neural Network for Stable Path Tracking of Mobile Robots: Adaptive learning rates approach," IEEE Trans. Circuit Syst., 2006, 53(6): 1381 1394.

[10] F. J. Lin, R. J. Wai, and C. C. Lee, "Fuzzy Neural Network Position Controller for Ultrasonic Motor Drive Using Push-pull DC-DC Converter,” in Proc. Inst. Elect. Eng.-Control Theory Appl., 1999, 146(1) : 99 107.

[11] C. Ye, N. H. C. Yung, and D. Wang, "A Fuzzy Controller With Supervised Learning Assisted Reinforcement Learning Algorithm for Obstacle Avoidance," IEEE Trans. Syst., Man, Cybern. B, Cybern., 2003, 33 ( 1): 17-27.

[12] R. J. Wai and C. C. Chu, "Motion Control of Linear Induction Motor via Petri Fuzzy-neural-network," IEEE Trans. Ind. Electron., 2007, 54 (1): 281 295.

[13] Tang Xu-Dong, Pang Yong-Jie, Zhang He, Lu Guo-Chun. Improved PSO-based S Plane Controller for Motion Control of Underwater Vehicle. 2008 Chinese Control and Decision Conference, Yantai, Shandong, China. 2008.7 : 2843 2848

[14] Wang Jian-Guo, Wu Gong-Xing, Wan Lei, Sun Yu-Shan, Wang LiRong. Controller Design of Underwater Robots Based on Generalized S-plane. Electric Machines and Control. 2009, 13(1): $144 \sim 148$
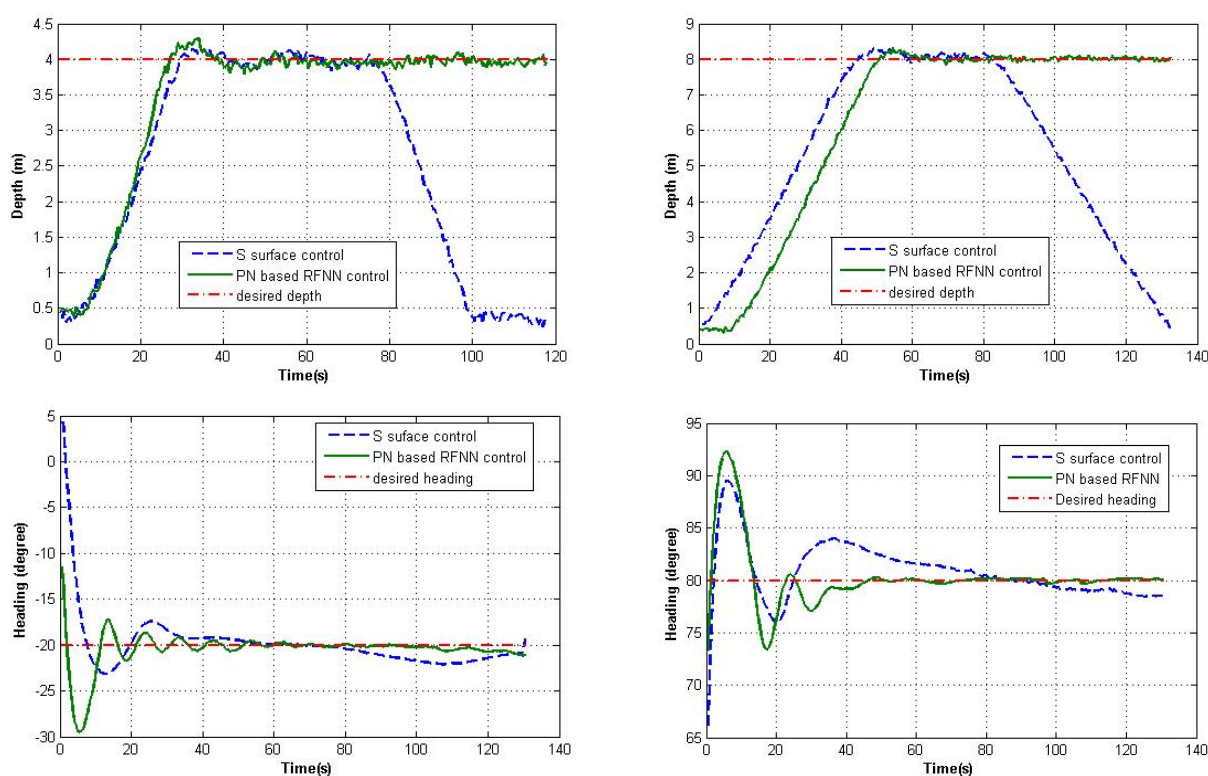

Figure 6. Experimental results and comparisons 